\title{
Keratoacanthomas following definitive volumetric modulated arc radiotherapy for skin field cancerization
}

\begin{abstract}
We describe three cases of keratoacanthomas (KAs) following definitive volumetric modulated arc therapy (VMAT) for skin field cancerisation (SFC). One lesion arose on a back in an area that had received a total of 30.6 Gy in 17 fractions. It first became apparent at 24 days post RT and resolved without treatment by 150 days. The other arose on a nasal alar in the beam penumbra that received 36Gy in 25 fractions, appearing at 57 days and resolving without treatment by 85 days. A third case occurred in an immunosuppressed patient. She developed KAs in one of two areas irradiated with lesions appearing at 21 days post RT and finally resolving at 300 days despite acitretin. The area received a total dose of $41.4 \mathrm{~Gy}$ in 23 fractions. Spontaneous resolution of index KA confirmed the diagnosis in all cases. On average, the patients were 79 years of age, the area bearing the KA had received an average of 36Gy in 22 fractions. The average onset of the KAs was 24 days or 3.5 weeks or one month after RT, and always soon after reepithelization was completed. Resolution was at an average of 168 days or 24 weeks or 6 months after the completion of RT. In all three cases other lesions arose adjacent to them and, in each case, one of these other lesions was excised with histopathology confirming squamo-proliferative lesions. There has been no recurrence at least 6 months after the last follow up. To our knowledge these are the first three cases of KA described following definitive VMAT for SFC. The possibility of KA arising after RT does need to be considered during the consent process.
\end{abstract}

Keywords: keratoacanthoma, radiotherapy, skin cancer, case study, volumetric modulated arc therapy (VMAT), skin field cancerisation, Australia, squamous cell carcinoma, immunosuppression
Volume 6 Issue 6 - 2019

SJ Mullin, ' A Lochhead, ${ }^{2}$ R Haddad, ${ }^{3}$ J Kippen, ${ }^{4} \mathrm{~V}$ Paddon, ${ }^{5} \mathrm{~A}$ Joshua, ${ }^{5,6}$ Karen Koh, ${ }^{7}$

Andrew Potter, ${ }^{8}$ GB Fogarty $1,5,9$

'School of Medicine, University of Notre Dame Australia,

Australia

${ }^{2}$ SydPath, St Vincent's Hospital, Sydney, Australia

${ }^{3}$ St Vincent's Private Hospital, Sydney, Australia

${ }^{4}$ Sydney Adventist Hospital Clinic, Sydney, Australia

${ }^{5}$ St Vincent's Hospital, Sydney, Australia

${ }^{6}$ Kinghorn Cancer Centre, Sydney, Australia

${ }^{7}$ Adelaide Skin \& Eye Centre, Australia

${ }^{8}$ GenesisCare, 352 South Terrace, Adelaide SA 5000,Australia

'GenesisCare, St Vincent's Hospital,Victoria Street, Darlinghurst, NSW 2010 Australia

Correspondence: Gerald B Fogarty, GenesisCare, St Vincent's Hospital, 438Victoria Street, Darlinghurst NSW 2010,Australia, Tel +6I 28302 5400, Fax +6I 283025410 ,

Email Gerald.Fogarty@genesiscare.com

Received: November 24, 2019 | Published: December 09, 2019

\section{Introduction}

The rates of skin cancer such as cutaneous squamous cell carcinomas (cSCC) and basal cell carcinomas (BCC) are increasing globally ${ }^{1,2,3}$ especially in Australia. Skin field cancerisation (SFC) describes large areas of in-situ disease from which invasive lesions can arise. ${ }^{3}$ Treatment of SFC can decrease the risk of subsequent invasive skin cancer, ${ }^{3-6}$ thereby avoiding the risk of spread and the need for subsequent lesion-focussed treatments with the consequent impact on quality of life and cost. ${ }^{7}$ Current treatments of SFC such as topical 5-fluorouracil, imiquimod, ingenol mebutate and diclofenac gel, photodynamic therapy (PDT), and surgery are disappointing, $, 3,6,8,9$ Radiotherapy (RT) can be used for treating invasive and in-situ or preinvasive skin cancer. ${ }^{8}$ Advances in RT, especially volumetric modulated arc therapy (VMAT), ${ }^{8,9,10}$ have enabled the delivery of highly conformal and homogenous radiation to large convex volumes like those usually involved in $\mathrm{SFC}^{1,6,10}$ with reduced treatment times and greater sparing of normal tissue. ${ }^{11-14}$ We report for the first time three cases of keratoacanthomas (KAs) arising in the regions treated with VMAT for SFC. In all cases the KAs arose in areas receiving less than the prescription dose.

\section{Case one}

A well, immunocompetent 84-year-old Caucasian male presented with extensive SFC of his back from decades of sun exposure (Figure
1). He had had multiple excisions for invasive skin cancers from his back, increasingly over the previous 30 years. The convalescence from each surgery was interfering with his quality life and he sought a non-surgical option.

\section{Treatment prescription and progress}

He was treated according to our national VMAT for SFC protocol. ${ }^{1}$ In summary, the treatment area measured $40 \mathrm{cmx} 40 \mathrm{~cm}$ (Figure 1) and was covered with $1 \mathrm{~cm}$ Superflab ${ }^{\circledR}$ (Radiation Products Design Inc, Albertville MN) bolus during treatment to ensure full dose on skin. Prescription dose was 45 Gy in 25 fractions, 1.8 Gy daily (MondayFriday) with VMAT via two arcs using 6MV RT (Figure 2). In vivo dosimetry on skin showed the planned dose was delivered (Table 1). An optional break at 10 fractions was declined. (See figure $3 \mathrm{~A}-\mathrm{N}$ for the evolution of acute reaction and KAs over time). After the $17^{\text {th }}$ treatment, at $30.6 \mathrm{~Gy}$, (Figures $3 \mathrm{~A}-\mathrm{D}$ ) the patient experienced considerable pain associated with brisk erythema and moist desquamation. Treatment was ceased.

By 16 days after RT cessation the back had re-epithelised (Figures $3 \mathrm{E} \& \mathrm{~F}$ ). At 24 days tender keratoacanthoma-type lesions were observed arising on the patients back within the treatment field, causing new pain (Figure 3G). At 54 days a large KA on the lower left back reached its maximum diameter (Figure $3 \mathrm{~K}$ ) and began to involute. A referral was made for excisional biopsy of other lesions. 
At 96 days post RT a lower right lesion was surgically removed-see histopathology in Figure 4. At 150 days post RT the index KA had fully resolved. No lesion has since recurred locally or regionally after 10 months of follow up. At 96 days post radiotherapy excision biopsy histopathology of the lower right lesion indicated in $3 \mathrm{M}$ revealed a squamous proliferative lesion. Parts of the lesion resembled KA, elsewhere the tumour showed features of well differentiated SCC (Figure 4).

Table I In vivo dosimetry on skin showed the planned dose was delivered, especially to lower back

\begin{tabular}{lllll}
\hline Location description & Detector position & Fraction 19 dose(Gy) & Total treatment dose (Gy) & \% of prescription \\
\hline Upper left back & A & 1.66 & 41.4 & $92 \%$ \\
Upper right back & B & 1.72 & 42.9 & $95 \%$ \\
Mid back & C & 1.82 & 45.5 & $101 \%$ \\
Lower left back & D & 1.8 & 44.9 & $100 \%$ \\
Lower right back & E & 1.8 & 45 & $100 \%$ \\
\hline
\end{tabular}

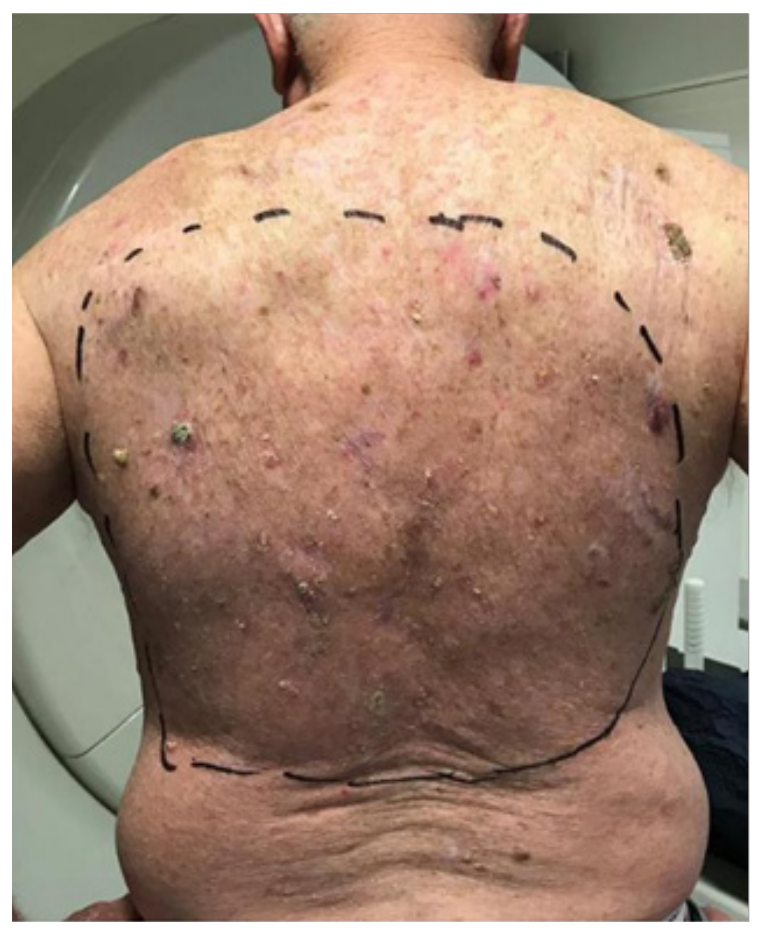

Figure I SFC treatment area measured $40 \mathrm{~cm} \times 40 \mathrm{~cm}$.
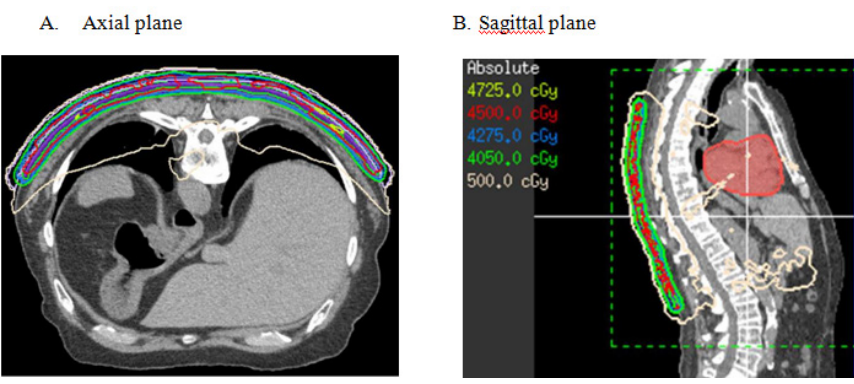

Figure 2 Radiotherapy planning scans showing dosimetry in the axial $(\mathrm{A})$ and sagittal (B) planes.

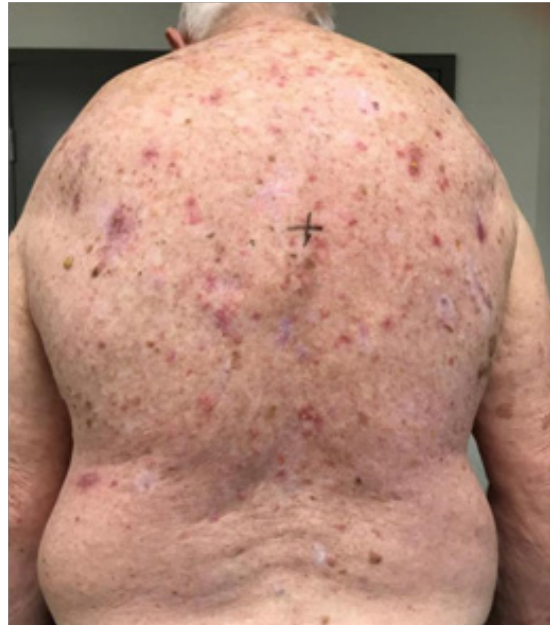

A.At $9 \mathrm{~Gy} / 5 \# \mathrm{~s}$

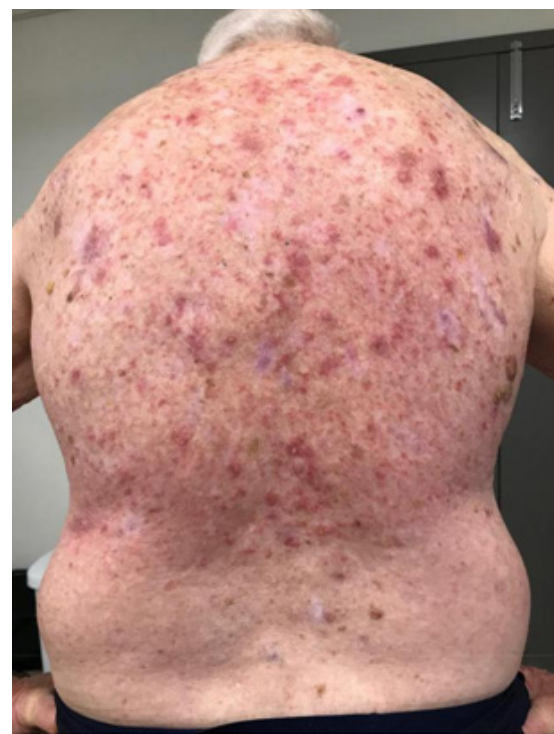

B.At $16.2 \mathrm{~Gy} / 9 \#$ s. 


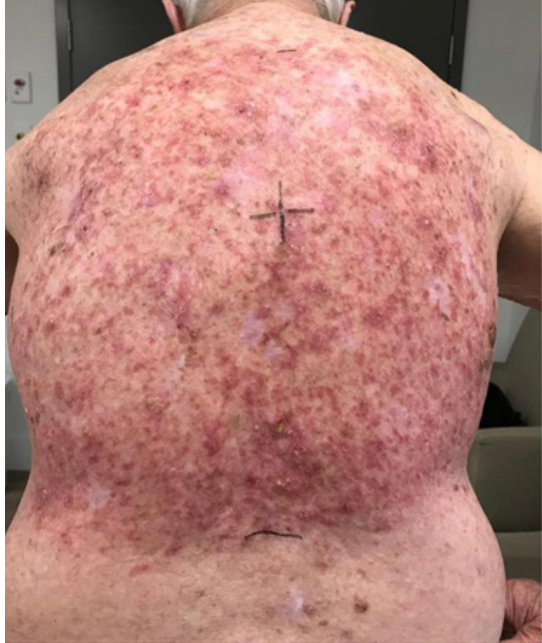

C.At $25.2 \mathrm{~Gy} / \mathrm{I} 4$ \#s.

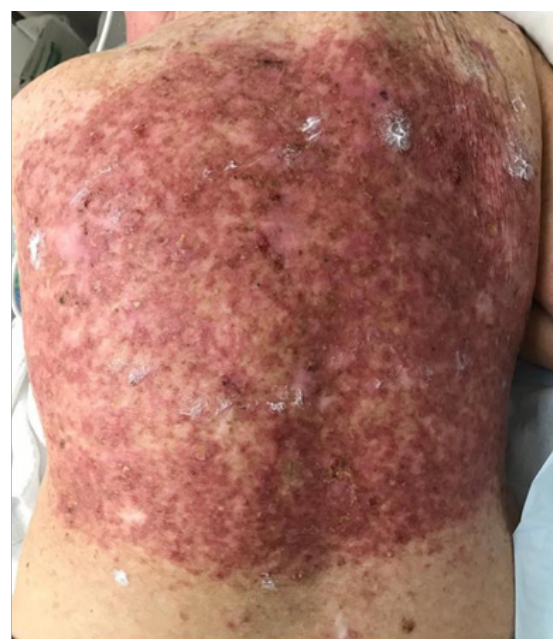

D.At 30.6Gy/ I7\#s - RT stopped due to pain.

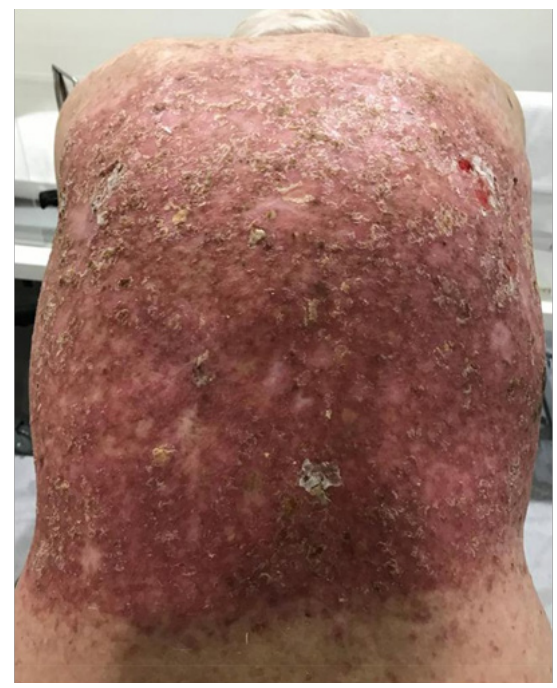

E. 6 days after $30.6 \mathrm{~Gy}$

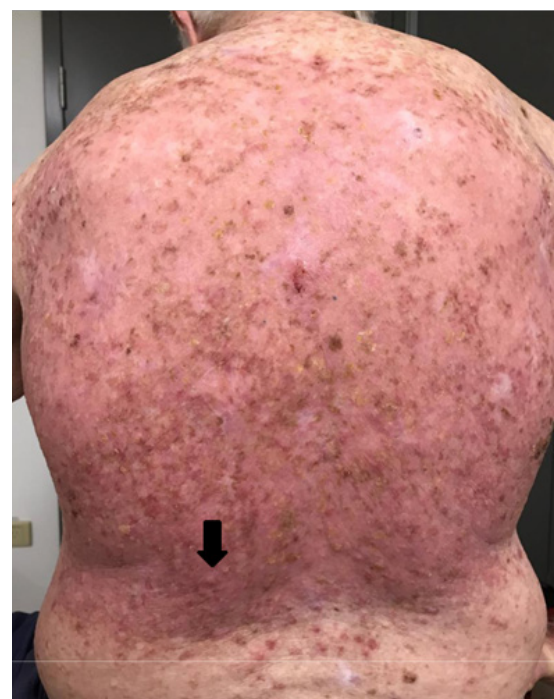

F. 16 days post RT - Re-epithelisation was complete. Black arrow throughout the remaining figures shows evolving KA that spontaneously resolves over the next 135 days.

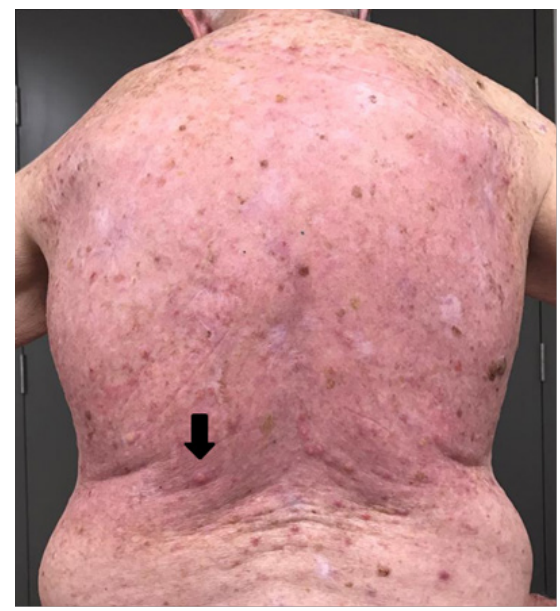

G. 24 days post RT - keratoacanthoma-type lesions were observed arising on the patients back within the treatment field.

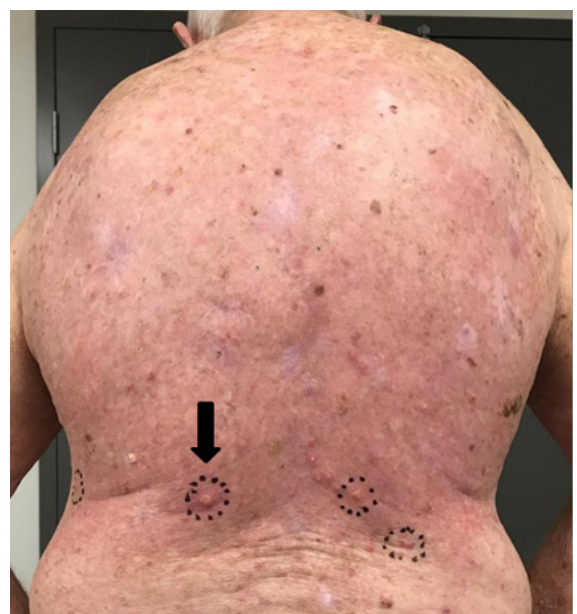

H. 33 days post RT - KAs continue to arise in field. 


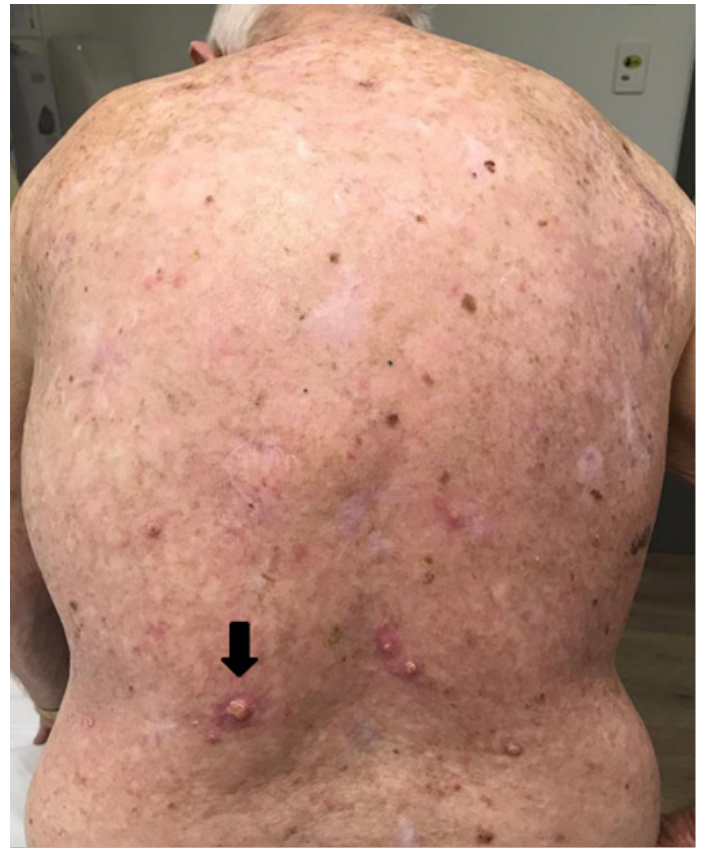

\section{47 days post RT.}

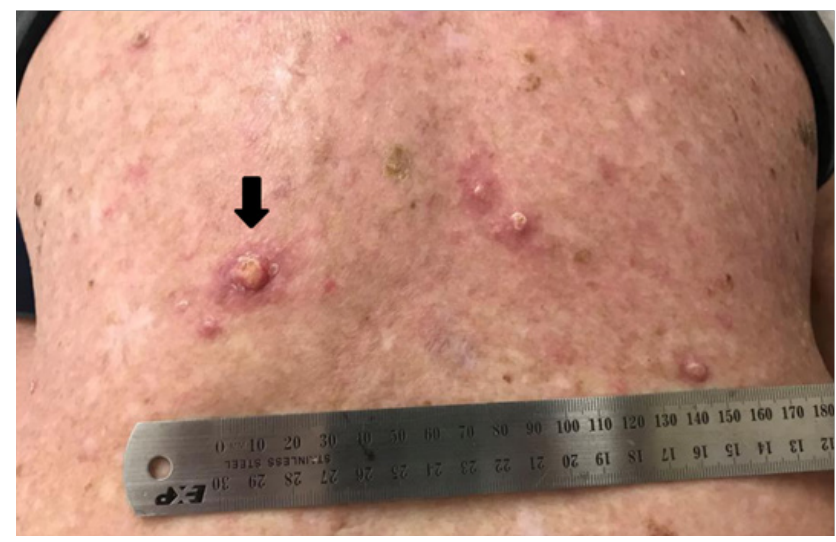

J. 47 days post RT - Close up.

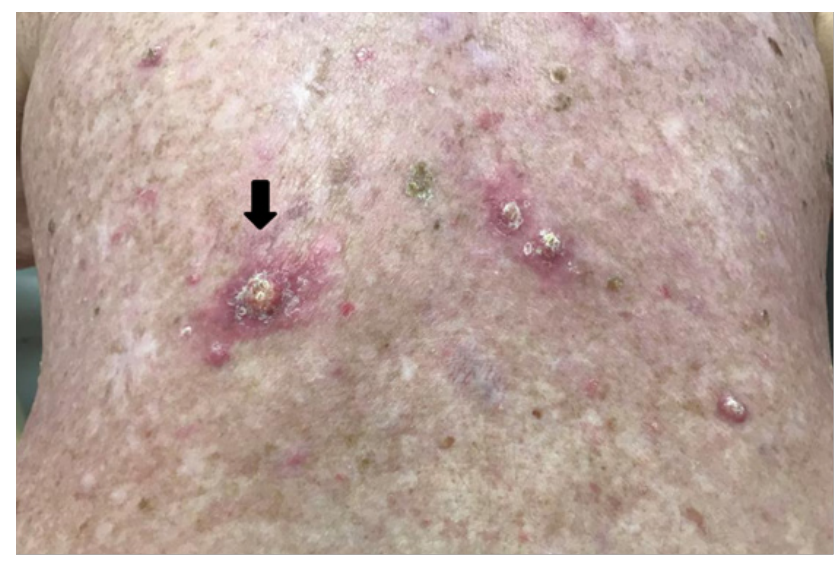

K. 54 days post RT close up.

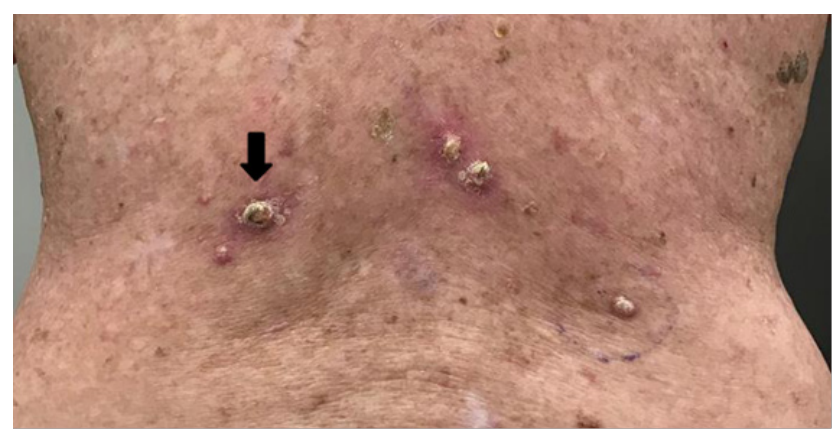

L. 68 days post RT - close up.

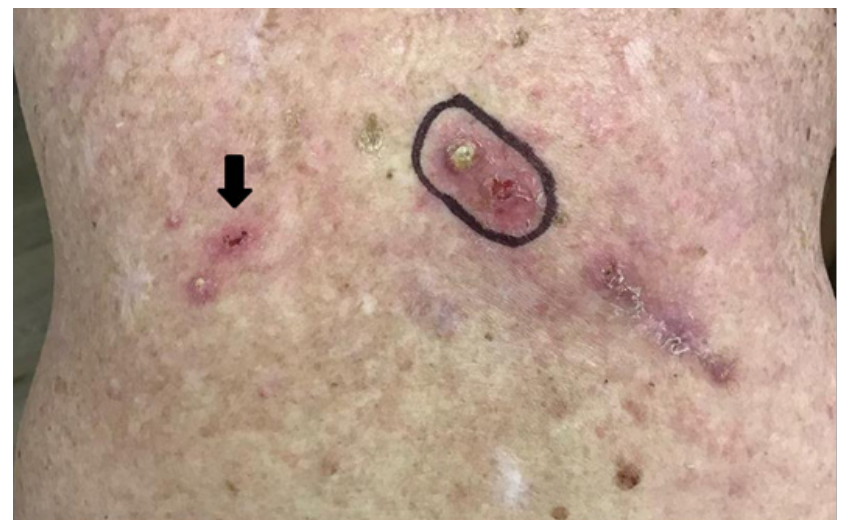

M. 96 days post RT.

Note surgical removal of lower right lesion - see histopathology in Figure 4, Note spontaneous regression of large left lesion.

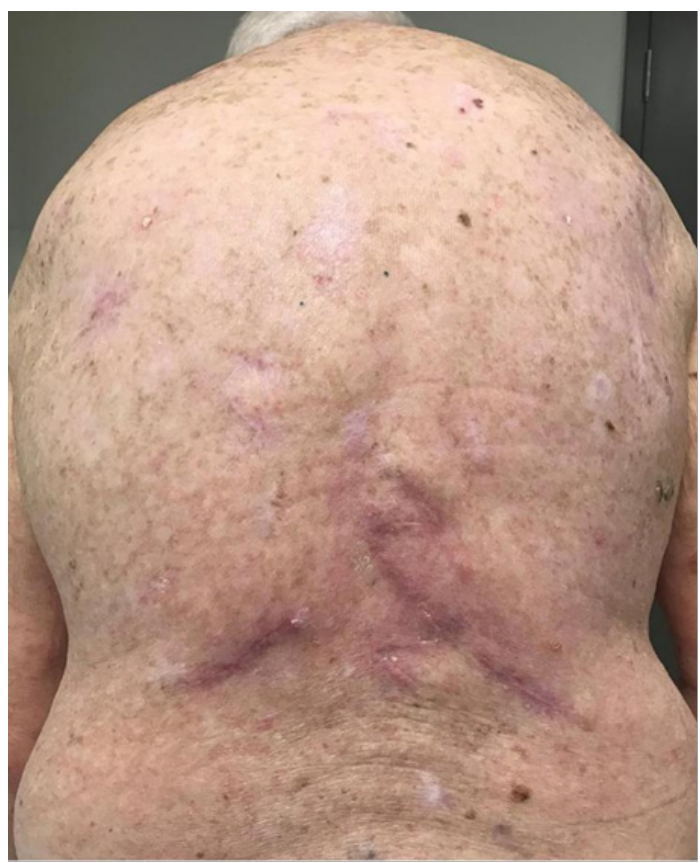

N. I 50 days post RT following three episodes of surgery - complete resolution of the untreated index KA confirming KA status.

Figure 3 (A-N) Progress of RT reaction on back. 


\section{A. Low power}

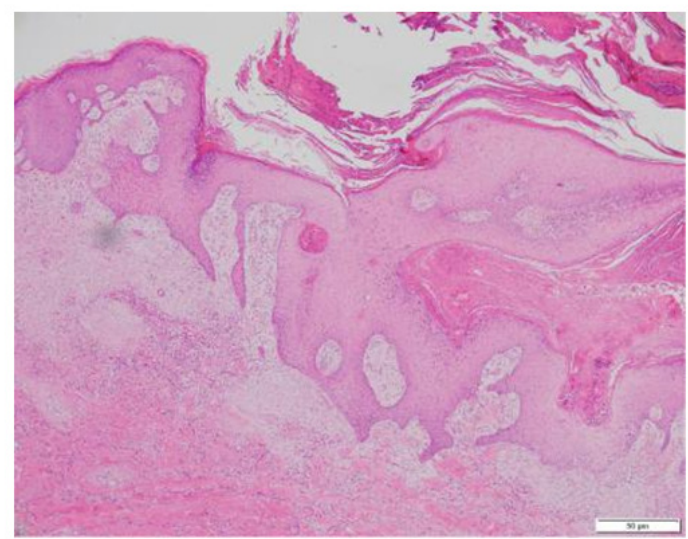

\section{B. High power}

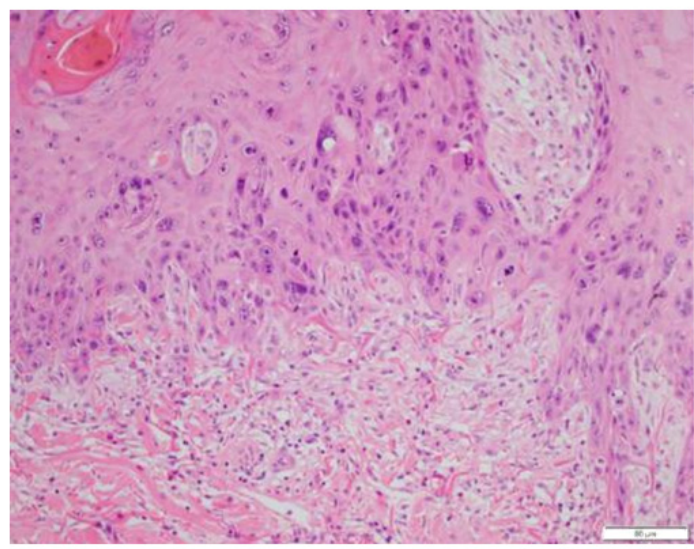

Figure 4 Histopathology of excision biopsy 96 days post RT.

\section{Case two}

A well, immunocompetent 81-year-old Caucasian male presented with a lesion on the tip and bridge his nose which was confirmed on punch biopsy as moderately differentiated SCC invading the dermis to a depth of $1 \mathrm{~mm}$. The nose had previous surgery for BCC many years prior and the current SCC arose from under the flap of this previous surgery. The patient was not keen on further surgery. The patient was considered suitable for curative intent VMAT therapy and he consented to RT.

\section{Treatment prescription and progress}

The patient tolerated the prescribed treatment of $55 \mathrm{~Gy}$ in 25 fractions daily (Monday-Friday) over five weeks using $6 \mathrm{MV}$ as per our published technique ${ }^{1}$ and case study. ${ }^{14}$ Figure 5 shows the extent of the RT field on the left side of nose at simulation. In vivo dosimetry showed the planned dose was delivered. At follow up 28 days post treatment cessation, two non-tender lesions were observed arising in the penumbra of the treatment volume. From the RT planning studies these lesions arose in areas of skin in the penumbral region that received 43 and 36 Gy respectively. The lesion that received 43 Gy was excised and was reported as a cystic, well-differentiated squamoproliferative lesion, resembling a trichilemmal cyst. The other lesion that had received 36 Gy behaved in a KA manner, resolving without treatment over weeks as illustrated by Figure $6 \mathrm{~A}$ and B. Neither lesion has recurred locally nor regionally at last follow-up which was 6 months post RT.

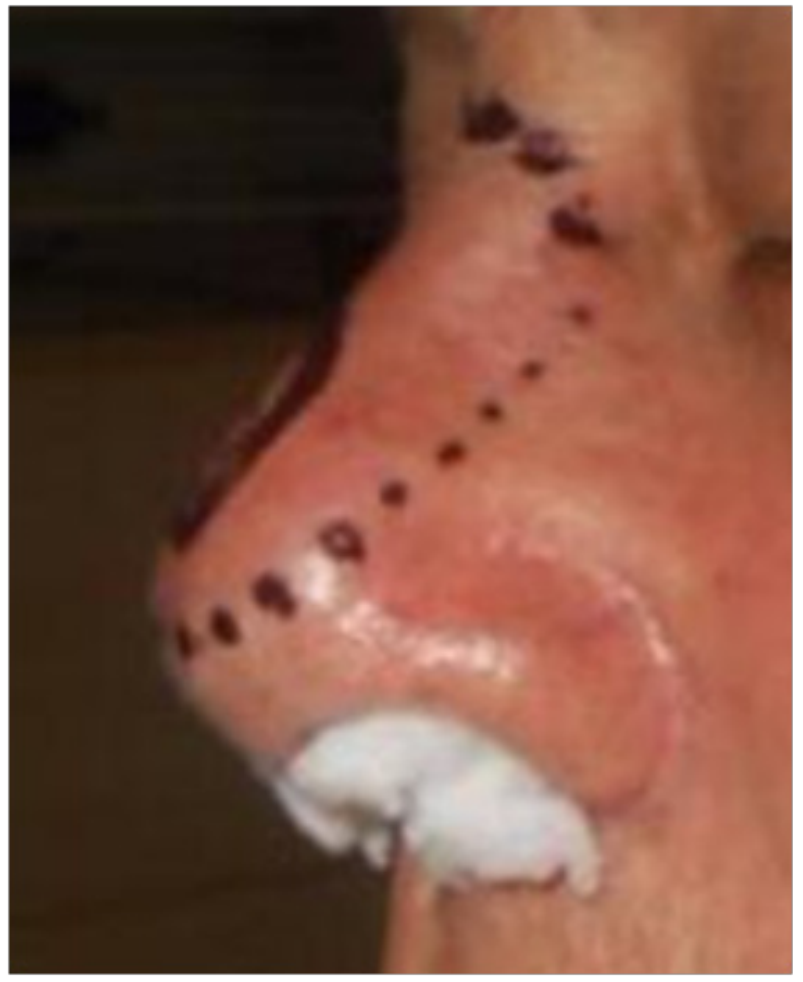

Figure 5 Extent of the RT field on the side of nose at simulation

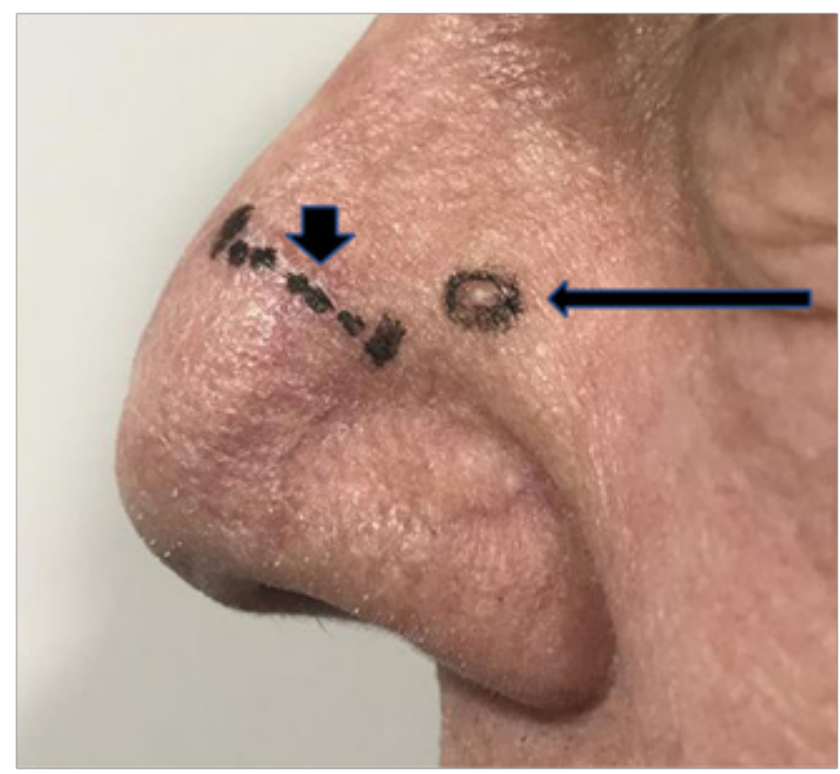

A. Lateral nose 57 days after RT. Photo shows scar marked with short vertical black arrow where a lesion that received 43 Gy had been removed at 36 days post RT in an area. The index KA marked by long horizontal black arrow is progressing in an area that received $36 \mathrm{~Gy}$. Comparison to figure 5 shows that this lesion is arising outside the area planned to receive prescription dose. 


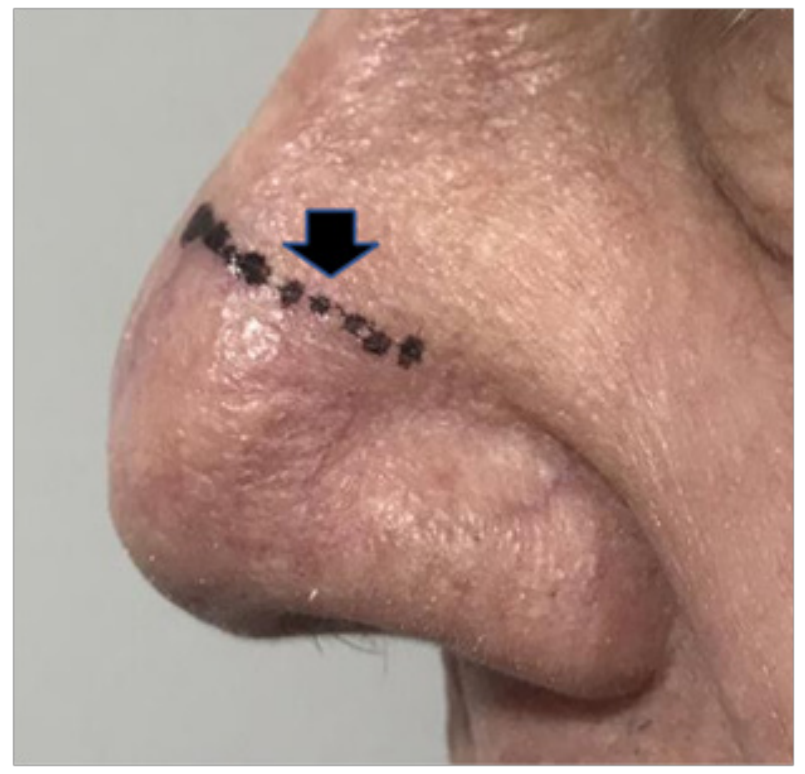

B. Lateral nose 85 days after RT. Photo shows scar marked with short vertical black arrow. Lesion in $36 \mathrm{~Gy}$ zone has now spontaneously resolved confirming KA status.

Figure 6 Lateral nose 57 days after RT.

\section{Case three}

A 72-year old Caucasian female was referred for consideration of radiotherapy for extensive SFC involving bilateral upper and lower limbs. She was immunosuppressed on the basis of liver transplantation 23 years earlier requiring long-term immunosuppression since then. Azathioprine had been ceased on account of progressive skin lesions, but she continued on cyclosporin. Multiple keratinocyte skin cancers had been previously excised, and previous treatments for SFC had included topical 5FU and cryotherapy without lasting benefit. She wished to avoid further surgical intervention and was assessed as suitable for VMAT radiotherapy for SFC.

\section{Treatment prescription and progress}

Treatment was prescribed in accordance with our published technique ${ }^{1}$, to a dose of $45 \mathrm{~Gy}$ in 25 fractions to the right lower limb, and $45 \mathrm{~Gy}$ in 25 fractions to the right forearm (including a simultaneous integrated boost to a clinically suspected squamous cell carcinoma on the mid-forearm). Both regions were treated simultaneously five days per week, with a planned 2-week break after 12 fractions). In vivo dosimetry confirmed that the delivered dose was within tolerance of the treatment plan. The patient tolerated the prescribed treatment to the upper limb well.

She developed significant acute lower leg pain and progressive oedema after 14 fractions (25.2 Gy), two fractions after the planned break, with patchy moist desquamation. Ultrasound excluded a deep vein thrombosis. With appropriate analgesia and skin care, treatment continued to a total dose of $41.4 \mathrm{~Gy}$ in 23 fractions before being ceased due to increasing pain. Symptoms improved significantly within 10 days of completing radiotherapy, with evidence of resolving field changes in both the upper and lower limb.

On review 42 days after treatment, the field changes in the upper limb had resolved, with no evidence of residual keratoses. However, in the lower limb the patient reported multiple rapidly enlarging, tender, hyper-keratotic lesions emerging throughout the irradiated lower limb field (Figure 7) and beyond. These were first noted approximately 21 days after completion of radiotherapy and had increased in size and number in the following weeks. Clinically the lesions resembled KAs, and the diagnosis was verified clinically by the patient's dermatologist. A dominant persisting lesion on the foot was excised with histopathology confirming a hyperkeratotic squamo-proliferative lesion in keeping with keratoacanthoma. This lesion was outside the RT field. No such lesions were identified within the treated upper limb field. The patient was commenced on oral acitretin $10 \mathrm{mg}$ alternate days, approximately 8 weeks after radiotherapy. The medication was well tolerated and the majority of lesions became crusted, indurated and regressed. The dose of acitretin was increased to $10 \mathrm{mg}$ daily after several months but was ceased due to dry eyes and lips. A number of small KA lesions were observed to persist 6 months after completion of radiotherapy, and her acitretin dose was restarted at the alternate day dose, increased to $10 \mathrm{mg}$ daily, before being ceased due to mild cognitive impairment. Complete resolution finally occurred 300 days (10 months) after completion of radiotherapy. These case finding are summarised in Table 2.
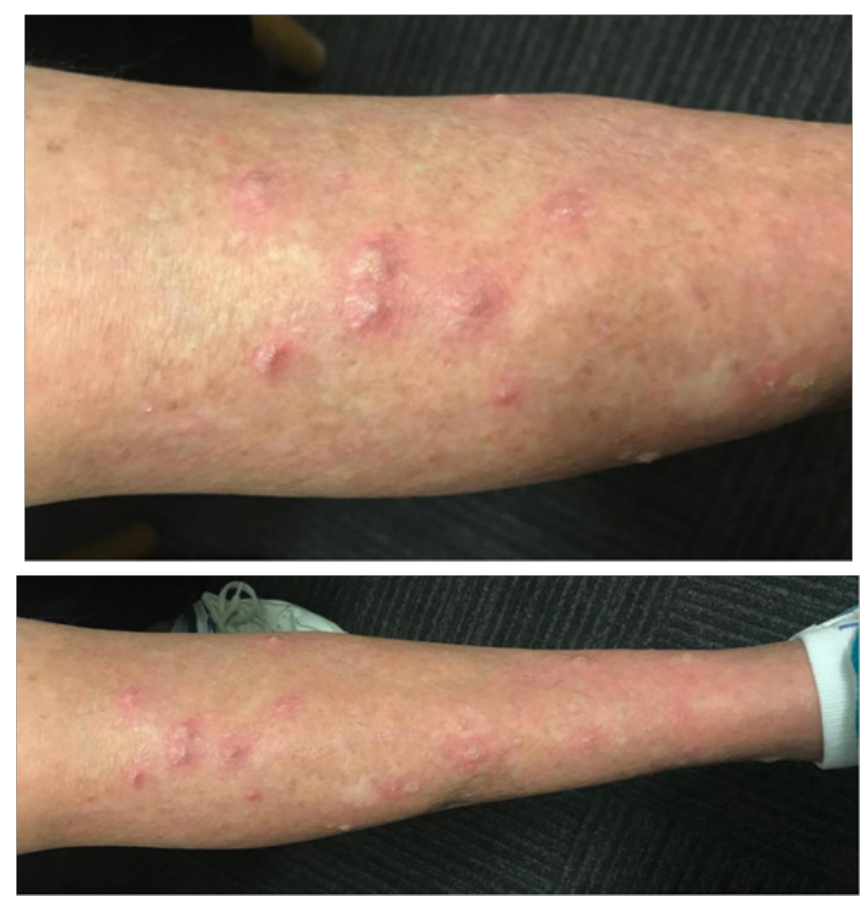

Figure 7 Lesions arising in the lower leg within the VMAT field. The upper photo is a close up.

\section{Discussion}

We report three cases of KA following completion of VMAT for SFC. One lesion arose on a back in a large area that had received 30.6 Gy in 17 fractions. It first became apparent at 24 days post RT and resolved by 96 days. The other arose in a small area on a nasal alar in the beam penumbra that received 36Gy in 25 fractions, first noticed at 57 days and resolving by 85 days. The third arose in an immunosuppressed patient in the leg in a large area that was irradiated to 41.4 Gy in 23 fractions. These KAs resolved spontaneously. Other lesions arose adjacent to them that were excised and histopathology 
revealed squamo-proliferative lesions. These were possibly also KAs, but as they were excised, spontaneous resolution, so typical and confirmatory of KA, was not observed, and so their status as KAs cannot be confirmed. No lesions have recurred locally or regionally at least 6 months after the last follow up. There have been previously published reports of KAs arising following skin cancer treatment There has been one other published report of KAs arising following RT. ${ }^{15}$ This case differs from our series in that it was with older RT techniques, was for lesion-based treatment for cSCC rather than fieldbased treatment and occurred in a patient with a personal and family history of multiple KAs. ${ }^{15}$

Table 2 Characteristics of KAs post definitive VMAT for SFC

\begin{tabular}{lllllll}
\hline $\begin{array}{l}\text { Case } \\
\text { number }\end{array}$ & $\begin{array}{l}\text { Age(yrs)/ } \\
\text { sex }\end{array}$ & $\begin{array}{l}\text { Immune- } \\
\text { suppressed }\end{array}$ & $\begin{array}{l}\text { Site/body zone/size of } \\
\text { SFC/tender }\end{array}$ & $\begin{array}{l}\text { Dose/fraction } \\
\text { Delivered } \\
\text { (Gy/\#) }\end{array}$ & $\begin{array}{l}\text { Days post } \\
\text { RT to } \\
\text { onset }\end{array}$ & $\begin{array}{l}\text { Days post } \\
\text { RT to } \\
\text { resolution }\end{array}$ \\
\hline 1 & $84 \mathrm{M}$ & No & $\begin{array}{l}\text { Back/trunk/large/tender } \\
\text { Nose/H+N*/Small/non- }\end{array}$ & $30.6 / 17$ & 24 & 150 \\
2 & tender & $36 / 25$ & 28 & 85 \\
3 & No & Leg/limbs/large/tender & $41.4 / 23$ & 21 & 300 \\
Average & 79 & & & $36 / 22$ & $\begin{array}{l}24 \text { or } 3.5 \\
\text { weeks or } \\
\text { one month }\end{array}$ & $\begin{array}{l}178 \text { or } 25 \\
\text { weeks or } 6 \\
\text { months }\end{array}$ \\
\hline
\end{tabular}

\section{$* \mathrm{H}+\mathrm{N}=$ head and neck}

Reports of KAs arising following SFC treatment with other modalities include reports following PDT, ${ }^{16-18}$ imiquimod, ${ }^{19,20}$ and surgery, with and without skin grafts. ${ }^{21}$ Provoking factors for KA include immunosuppression or immunodeficiency, trauma, surgery, irritation after topical drugs (imiquimod), systemic chemotherapies, microdermabrasion, chemical peels, laser therapy and electromagnetic radiation. ${ }^{16}$ The usual treatment for KA is surgical excision driven mainly by a concern that the KA may progress to invasive SCC rather than spontaneously regress. Our three cases had surgery to nearby lesions that may have been KAs. Surgery may not always be feasible. Treatment with oral acitretin has been used successfully in cases not amenable to surgery ${ }^{15}$ as has intralesional 5-FU, methotrexate, imiquimod, PDT, laser therapy and RT. ${ }^{22,23}$ There are several interesting radiobiological aspects to these cases. They arose in areas that did not receive the prescription dose. They arose in all possible VMAT in SFC zones i.e., head and neck, trunk and limbs. They arose within weeks after re-epithelisation of the irradiated area had been completed. The larger lesions were tender. Was this a function of size only?

There are also several interesting immunological aspects to these cases. In the immunosuppressed patient the KAs appeared at the same time as in the immune competent but took longer to resolve despite acitretin. In the same patient, KAs arose in the leg but did not arise in the treated area of the arm, which was treated at the same time to the same dose. The cause of the KAs in the leg also cannot then be solely due to immunosuppression. These observations also suggest that genetic factors alone are not the cause, and a local factor may be involved. KAs also appeared outside the field, more specifically in the foot below the leg field. Was this a function of the acute radiation oedema travelling to dependent sites taking factors that caused KAs to arise out of field? These are all hypothesis-generating observations. More study especially with translational end points is needed.

In these early days of VMAT for SFC it is important to describe unusual findings. Involvement of a multidisciplinary team may help guide the appropriate treatment for this phenomenon. Radiation oncologists may now include the possibility of KAs arising after
VMAT for SFC in the consenting process. The degree to which this phenomenon is emphasised in that process will be made clearer when further studies are completed, especially studies that will measure the actual incidence of KAs arising after VMAT for SFC over the denominator of total cases, the vast majority of which have not experienced KAs.

\section{Conclusion}

The first cases of KAs following VMAT for SFC are reported. In these three cases, the KA's appeared in areas treated with less than the prescription RT dose and as such did not receive full therapeutic RT for SFC. KA needs to be recognised as a possible toxicity in this scenario. More studies are needed.

\section{Funding}

None.

\section{Ethical consent}

We are grateful to the patients concerned for allowing us to publish their clinical outcomes and photos.

\section{Conflicts of interest}

Author declares that there is no conflict of interest.

\section{Acknowledgments}

GenesisCare at St Vincent's Hospital, Sydney and South Terrace, Adelaide.

\section{References}

1. Potter A, Price M, Papworth D, et al. A technique for treating extended skin field cancerisation using volumetric modulated arc therapy. Int $J$ Radiol Radiat Ther. 2019;6(4):111-119.

2. Apalla Z, Nashan D, Weller RB, et al. Skin cancer: epidemiology, disease burden, pathophysiology, diagnosis, and therapeutic approaches. Dermatol Ther (Heidelb). 2017;7(Suppl 1):5-19. 
3. Christensen SR. Recent advances in field cancerization and management of multiple cutaneous squamous cell carcinomas. F1000Res. 2018;7.

4. Torezan LA, Festa-Neto C. Cutaneous field cancerization: clinical, histopathological and therapeutic aspects. An Bras Dermatol. 2013;88(5):775-786.

5. Lanoue J, Chen C, Goldenberg G. Actinic keratosis as a marker of field cancerization in excision specimens of cutaneous malignancies. Cutis. 2016;97(6):415-420.

6. Fogarty GB, Christie DRH, Kaminski A, et al. A radiation oncology approach for using definitive radiotherapy with volumetric modulated arc therapy (VMAT) for skin field cancerisation (SFC). Int J Radiol Radiat Ther. 2018;5(4):227-234.

7. Gaulin C, Sebaratnam DF, Fernández-Peñas P. Quality of life in nonmelanoma skin cancer. Australas J Dermatol. 2015;56(1):70-76.

8. Hofbauer G, Anliker M, Boehncke WH, et al. Swiss clinical practice guidelines on field cancerization of the skin. Swiss Med Wkly. 2014;144:w14026.

9. Chaplin S. Topical agents for preventing and treating actinic keratosis. Prescriber. 2016;27(4):32-40

10. Fogarty GB, Christie D, Spelman LJ, et al. Can Modern Radiotherapy be used for Extensive Skin Field Cancerisation: An Update on Current Treatment Options. Biomed J Sci \& Tech Res. 2018;4(1).

11. Teoh M, Clark CH, Wood K, et al. Volumetric modulated arc therapy: a review of current literature and clinical use in practice. $\mathrm{Br} J$ Radiol. 2011;84(1007):967-996.

12. Lozano F, Perez N, Iglesias A, et al. Volumetric arc therapy for total scalp irradiation: case report for a recurrent basal cell carcinoma of the scalp. Ecancermedicalscience. 2017;11:737.

13. Santos ED, Green JA, Bhandari A, et al. Tangential Volumetric Modulated Radiotherapy-A New Technique for Large Scalp Lesions with a Case Study in Lentigo Maligna. Int J Bioautomation. 2015;9(2):223-236.
14. Martin TD, Moutrie Z, Tighe D, et al. Volumetric modulated arc therapy (VMAT) for skin field cancerisation of the nose-A technique and case report. Int J Radiol Radiat Ther. 2018;5(3):142-148.

15. Robertson SJ, Bashir SJ, Pichert G, et al. Severe exacerbation of multiple self- healing squamous epithelioma (Ferguson-Smith disease) with radiotherapy, which was successfully treated with acitretin. Clin Exp Dermatol. 2010;35(4):e100-e102.

16. Kwiek B, Schwartz RA. Keratoacanthoma (KA): an update and review. $J$ Am Acad Dermatol. 20161;74(6):1220-1233.

17. Sarbu MI, Matei C, Mitran CI, et al. Photodynamic therapy: A hot topic in dermato-oncology. Oncol Lett. 2019;1;17(5):4085-4093.

18. Borgia F, Giuffrida R, Caradonna E, et al. Early and late onset side effects of photodynamic therapy. Biomedicines. 2018;6(1):12.

19. Campalani E, Holden CA. Keratoacanthoma associated with the use of topical imiquimod. Clin Exp Dermatol. 2013;38(5):555-556.

20. DAddario S, Carrington PR. Multiple keratoacanthomas as an untoward response to imiquimod therapy for actinic keratoses. Acta Derm Venereol. 2006;86(4):366-367.

21. Clark MA, Guitart J, Gerami P, et al. Eruptive keratoacanthomatous atypical squamous proliferations (KASPs) arising in skin graft sites. JAAD case rep. 2015;1(5):274-276.

22. Donahue B, Cooper JS, Rush S. Treatment of aggressive keratoacanthomas by radiotherapy. J Am Acad Dermatol. 1990;23(3 Pt 1):489-493.

23. Cerroni L, Kerl H. Chapter 117. Keratoacanthoma. In: Goldsmith LA, Katz SI, Gilchrest BA, Paller AS, Leffell DJ, Wolff K, editors. Fitzpatrick's Dermatology in General Medicine, 8e New York, NY: McGraw-Hill; 2012. 